\title{
Nodular lymphoid hyperplasia of urinary bladder admixed with non-invasive low-grade urothelial carcinoma
}

\author{
Tadashi Terada* \\ Department of Pathology, Shizuoka City Shimizu Hospital, Shizuoka, Japan
}

Received: February 28, 2016

DOI: $10.5430 /$ crcp.v3n2p64

\author{
Accepted: April 19, 2016 \\ Online Published: May 11, 2016 \\ URL: http://dx.doi.org/10.5430/crcp.v3n2p64
}

\begin{abstract}
Primary malignant lymphoma of urinary bladder is extremely rare, and benign nodular lymphoid hyperplasia has not been reported in bladder. Nodular lymphoid hyperplasia of bladder associated with bladder carcinoma has also not been reported. Herein the author describe a 64-year-old man who presented with dysuria and his cystscopic examination revealed a papillary tumor in bladder. A transurethral resection of bladder tumor was carried out, and histopathologic examination revealed a non-invasive pTa low-grade papillary urothelial carcinoma and nodular lymphoid hyperplasia that were mixed up. The nodular lymphoid hyperplasia was composed of inner germinal centers and outer marginal and mantle zones. The germinal centers were composed of tangible body macrophages, centrocytes and centroblasts, while the outer zone of mature lymphocytes. Immunohistochemical profile of nodular lymphoid hyperplasia was as follows: cytokeratin AE1/AE3-, cytokeratin CAM5.2-, CD45+++, CD20+++, CD79a++, CD138-, CD3-, CD45RO-, CD15-, CD30-, p53- Ki67 80\% (confined to germinal centers), bcl-2-, bcl-6++, CD10++, MUM1-, cyclinD1-, light chains-, CD56-, TdT-, CD68-. The patient is now followed up without any treatments by periodical cystscopy with BCG injection and cytology. No recurrence is noted 13 months after the first presentation. In conclusion, an extremely rare case of nodular lymphoid hyperplasia mixed with non-invasive urothelial carcinoma in bladder is reported.
\end{abstract}

Key Words: Bladder, Nodular lymphoid hyperplasia, Urothelial carcinoma

\section{INTRODUCTION}

Malignant lymphomas of urinary bladder are very rare. ${ }^{[1]}$ About one thirds of them are primary in urinary bladder and the remaining two thirds secondary. Almost all malignant lymphomas of bladder are of non-Hodgkin B-cell lymphoma. ${ }^{[1]}$ Primary malignant lymphomas of bladder are mostly marginal-zone B-cell lymphoma of mucosaassociated lymphoid system (MALT lymphoma). ${ }^{[1-4]}$ Diffuse large B-cell lymphomas primary in bladder have also been reported, though they are extremely rare. ${ }^{[2]}$ Reactive nodular lymphoid hyperplasia different from MALT lymphoma has not been reported, or its candidate has been reported only once by Schlager et $a l^{[5]}$ who reported 12 cases of nodular lymphoid hyperplasia of bladder associated bacterial cystitis and found that the lymphocytes were of B-cells. Nodular lymphoid hyperplasia of bladder must be differentiated from more common follicular lymphoma. The most common tumors of bladder are urothelial carcinoma, followed in order by adenocarcinoma and squamous cell carcinoma. Mixed carcinomas of these three types are relatively frequently seen. Nodular lymphoid hyperplasia associated with bladder cancers has not been reported in the English literature to the

*Correspondence: Tadashi Terada; Email: piyo0111jp@yahoo.co.jp; Address: Department of Pathology, Shizuoka City Shimizu Hospital, Miyakami 1231 Shimizu-Ku, Shizuoka 424-8636, Japan. 
best of the author's knowledge. Herein reported is an extremely rare case of nodular lymphoid hyperplasia of bladder admixed with non-invasive papillary low-grade urothelial carcinoma.

\section{CASE REPORT}

A 64-year-old man presented to our Urology Department with symptoms of dysuria. A cystoscopic examination revealed a papillary tumor of $11 \mathrm{~mm}$ in diameter in urinary bladder, and transurethral resection of bladder tumor was carried out. On gross inspection, the specimens consisted of many bladder fragments with a focus of papillary area. Eight sections were made, processed routinely, and stained with hematoxylin and eosin. A microscopical examination revealed a papillary epithelial tumor and nodular lymphoid proliferation that were mixed up. The latter was not discernible on gross inspection. The epithelial tumor was apparent noninvasive (pTa) papillary low-grade urothelial carcinoma (see Figure 1A). The urothelial carcinoma was judged by a mapping study to be completed resected with no tumor cells in the margins. The lymphoid proliferation was of nodular type (see Figure 1B) which showed polarity (see Figure 1C) and apparent tangible body macrophages (see Figure 1D). Each lymphoid nodule was composed of inner germinal center and outer marginal and mantle zones. The germinal centers were composed of tangible body macrophages, centrocytes and centroblasts, and the outer zone of mature lymphocytes (see Figure 1D). Neither lymphoepithelial lesions nor plasmacytosis were seen. No monocytoid B-cells were recognized. An Immunohistochemical study was carried out by Envision method, ${ }^{[6,7]}$ and the results were as follows: cytokeratin AE1/AE3-, cytokeratin CAM5.2-, CD45+++, CD20+++ (see Figure 1E), CD79a++, CD138-, CD3-, CD45RO-, CD15-, CD30-, p53- Ki67 80\% (confined to germinal center), bcl-2-, bcl-6++ (see Figure 1F), CD10++, MUM1-, cyclinD1-, light chains (both kappa and lamda)-, CD56-, TdT-, CD68-. Imaging techniques revealed no other tumors in the body (cM0, cNO). The pathological diagnosis of non-invasive low-grade pTa papillary urothelial carcinoma (pTa, cM0, cN0. Stage $0 \mathrm{~A})$ admixed with benign nodular lymphoid hyperplasia was made. According to NCI standard regimen of urothelial carcinoma of bladder at Stage 0A, the patient was treated by periodical cystscopy with BCG injection in the periods of 6 months; thereafter the patient is followed up by periodical cystscopy and clinical cytology. No recurrence or metastasis is found 13 months after the first presentation.

\section{DiscuSsion}

It is obvious that the present epithelial papillary tumor of bladder is non-invasive pTa papillary low-grade urothelial carcinoma. It is strongly suggested that the lymphoid lesion

Published by Sciedu Press is benign nodular lymphoid hyperplasia, but it must be differentiated from indolent B-cell neoplasms. The lymphoid proliferative lesion in this case was composed of nodular lesions resembling germinal centers and outer mature lymphocytic bands simulating germinal centers and marginal and mantle zones of normal lymph-node, respectively. The germinal center-like lesions of the present tumor consisted of tangible body macrophages, centrocytic cells and centroblastic cells, and the germinal center-like lesions and outer zones showed polarity of normal lymph nodes. These findings indicate that the lesion is not follicular lymphoma but benign germinal center. In contrast, the outer zones of the germinal centers in the present tumor were composed of mature lymphocytes resembling normal marginal and mantle zones, suggesting that the present tumor is not neoplastic. The Immunohistochemical findings also indicate that the present tumor is benign nodular lymphoid hyperplasia. The CD20 and CD79a indicate B-cell natures. The positive germinal cell antigens (bcl-6, CD10) and negative bcl-2 deny follicular lymphoma. Histological features and negative cyclin D1 deny mantle cell lymphoma. Histological features including negative lymphoepithelial lesions and negative plasm cells (CD138-negative) deny MALT lymphoma. Thus, the lesion was judged as nodular lymphoid hyperplasia. Of particular interest in the case is that the nodular lymphoid hyperplasia was associated with urothelial carcinoma. In general, tumor-infiltrating lymphocytes in tumor immunology are of T-cells. However, the present tumor was of B-cells, suggesting that B-cell immunity against urothelial carcinoma might be operative in the present case. Schlager et $a l^{[5]}$ also described predominant B-cells in nodular lymphoid hyperplasia of bladder caused by bacterial cystitis.

The present case is unique in that florid nodular lymphoid hyperplasia was seen in concomitant urothelial carcinoma in bladder. The fact in this case that the nodular lymphoid hyperplasia was composed almost of B-cells is also a unique aspect in the case. The author thinks that the treatment and prognosis are mainly associated with urothelial carcinoma rather than nodular lymphoid hyperplasia in the case. He also thinks that there are no significant clinical implications such as treatment and prognosis in nodular lymphoid hyperplasia in the case. Because, to the best of the author's knowledge, there are no reports of nodular lymphoid hyperplasia of bladder associated with urothelial carcinoma, the treatment of this combination case has not been investigated in the literature; and therefore it cannot be compared with previous reports. However, in the present case with such combination, only treatment for urothelial carcinoma (in the case, transurethral resection and BCG injection followed by periodical cystscopy and clinical cytology) seems to give 
rise to good results. There is no need for the use of antiCD20 antibody therapy (Rituximab). Low-grade urothelial carcinoma as seen in the case might have better prognosis in the case that high-grade carcinoma, being also true between non-invasive and invasive carcinoma; the present case is noninvasive. Finally, it must stressed that the present nodular lymphoid hyperplasia is different from MALT lymphoma histologically and immunohistochemically.

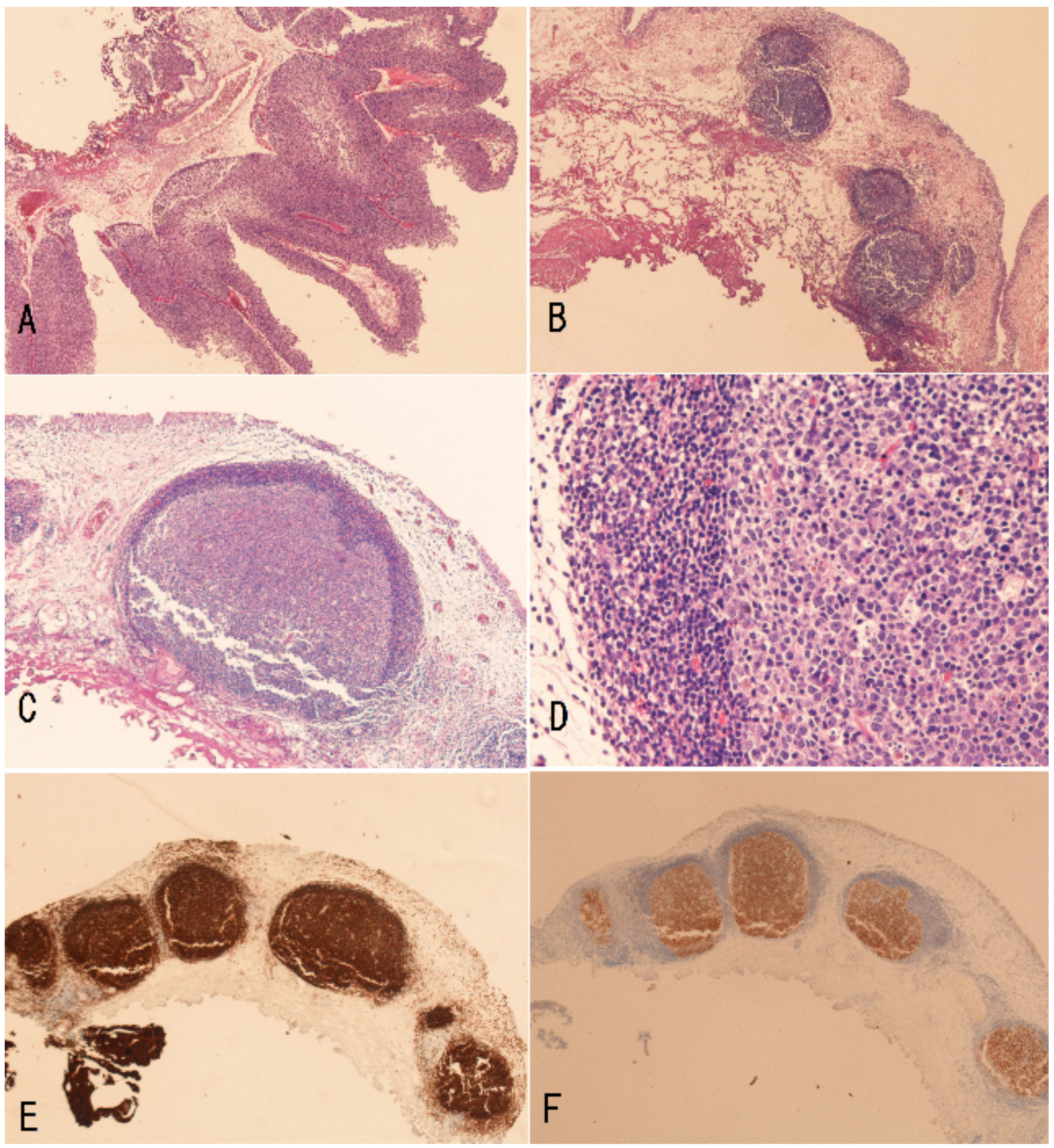

Figure 1. Histological (A-D) and Immunohistochemical (E,F) features of nodular lymphoid hyperplasia (B-F) and non-invasive low-grade urothelial hyperplasia (A) of urinary bladder

A: Low power view shows non-invasive low-grade urothelial carcinoma. $H E, \times 20$. B: Low power view shows nodular lymphoid hyperplasia. It is closely admixed with urothelial carcinoma, but it is not shown here. The nodular lymphoid hyperplasia is diffuse: only 4 nodular lesions are shown in this picture. HE, $\times 40$. C: Higher power view of nodular lymphoid hyperplasia shows one nodule with preservation of germinal center orientation. The lesion is composed of germinal center-like area and outer marginal and mantle zones. $H E, \times 150$. D: Much higher view shows the center of the lesion is composed of centrocytes, centroblast, and tangible body macrophages similarly to hyperplastic lymphoid nodules of lymph node. $H E, \times 250$. The nodular lymphoid hyperplasia shows CD20 positivity $(E)$ in diffuse areas and bcl-6 positivity $(F)$ in germinal centers. 


\section{Conclusion}

The first or second case of nodular lymphoid hyperplasia of urinary bladder is reported. The pathological features and differential diagnosis from indolent B-cell lymphoma are discussed. The present case is also unique in that the nodular lymphoid hyperplasia was mixed closely with non-invasive papillary urothelial carcinoma. B-cell immunity but not Tcell one seems to have effect against urothelial carcinoma in this case of nodular lymphoid hyperplasia.

\section{REFERENCES}

[1] Kempton CL, Kurtin PJ, Inwards DJ, et al. Malignant lymphoma of the bladder: evidence from 36 cases that low-grade lymphoma of the MALT-type is the most common primary bladder lymphoma. Am J Surg Pathol. 1997; 21: 1324-33. PMid: 9351570. http: //dx.doi.org/10.1097/00000478-199711000-00007

[2] Simpson WG, Lopez A, Babbar P, et al. Primary bladder lymphoma, diffuse large B-cell type: Case report and literature review of 26 cases. Urol Ann. 2015; 7: 268-72. PMid: 25837971. http://dx.doi.org/10.4103/0974-7796.152947

[3] Matsuda I, Zozumi M, Tsuchida YA, et al. Primary extranodal marginal zone lymphoma of mucosa-associated lymphoid tissue type with malakoplakia in the urinary bladder: a case report. Int J Clin Exp Pathol. 2014; 7: 5280-4. PMid: 25197410.

[4] Morita K, Nakamura F, Nannya Y, et al. Primary MALT lymphoma of the urinary bladder in the background of interstitial cys- titis. Ann Hematol. 2012; 91: 1505-6. PMid: 22297664. http: //dx.doi.org/10.1007/s00277-012-1419-0

[5] Schlager TA, LeGallo R, Innes D, et al. B cell infiltration and lymphonodular hyperplasia in bladder submucosa of patients with persistent bacteriuria and recurrent urinary tract infections. J Urol. 2011; 186: 2359-64. PMid: 22019034. http://dx.doi.org/10.1016 /j.juro.2011.07.114

[6] Terada T, Kawaguchi M. Primary clear cell adenocarcinoma of the peritoneum. Tohoku J Exp Med. 2005; 206: 271-5. PMid: 15942157. http://dx.doi.org/10.1620/tjem.206.271

[7] Terada T, Kawaguchi M, Furukawa K, et al. Minute mixed ductalendocrine carcinoma of the pancreas with predominant intraductal growth. Pathol Int. 2002; 52: 740-6. PMid: 12685552. http: //dx.doi.org/10.1046/j.1440-1827.2002.01416.x 